Mahantesh M. Nandeppanavar*, M. C. Kemparaju, and S. Shakunthala

\title{
MHD stagnation point slip flow due to a non-linearly moving surface with effect of non-uniform heat source
}

https://doi.org/10.1515/nleng-2017-0109

Received August 29, 2017; revised January 7, 2018; accepted February $10,2018$.

\begin{abstract}
In this paper, we have studied the heat transfer characteristics of stagnation point flow of an MHD flow over a non-linearly moving plate with momentum and thermal slip effects in presence of non-uniform heat source/sink. The governing differential equations are transformed into the ordinary differential equations using suitable similarity transformations. These equations which are BVP's and are solved using a numerically by fourth order Runge-Kutta method using MAPLE computing software. The effects of governing parameters are studied on flow, velocity and heat distributions and are discussed in detail. It is observed that the non-uniform heat source parameters enhance the temperature distribution. Our results are agreed well with previously published results for some limiting conditions, which validate our present results are correct.
\end{abstract}

Keywords: Stagnation point flow, MHD, non-uniform heat source/sink, moving plate, partial slip, Thermal jump

\section{Introduction}

As we know the present generations are very much depend on applications of science and technologies which are based on industries. In aerodynamic, extrusion of polymers, hot rolling, cooling of metallic plates, glass-fiber production etc. The magneto-hydrodynamic flow has both liquid as well as magnetic properties it can exhibits partic-

\footnotetext{
*Corresponding Author: Mahantesh M. Nandeppanavar, Department of Studies and Research in Mathematics, Government College, Gulbarga-585105, Karnataka, India, E-mail: mahantesh_maths@yahoo.co.in,mahantesh_maths@yahoo.co.in M. C. Kemparaju, Department of mathematics, Jyothy Institute of Technology, Bangalore-82

S. Shakunthala, Department of Studies and Research in Mathematics, Government College, Gulbarga-585105, Karnataka, India
}

ular characteristics in thermal conductivity. Raju et al. [1] considered a detail study of least square finite element on non-linear boundary layer problems analysed stagnation point flow. Gorla [2] has considered a non-Newtonian fluid of a stagnation point flow in the presence of the transverse magnetic field and resulted those shear stress co-efficient increases with increasing magnetic field strength for all the values of flow behavior index. Takhar et al. [3] studied MHD unsteady stagnation point boundary layer flow. Besser et al. [4] given a description of work of magnetic field annilation within the frame work of the MHD equations of an incompressible plasma's in the regions $y<0$ and $y>0$,along with the uniform resistivity and viscosity as a transport parameter. Massoudi and Ramezant [5] investigated heat transfer analysis of a viscoelastic fluid at a stagnation point. Donal [6] investigated the three dimensional stagnation point flow of a viscoelastic fluid. Mahapatra et al. [7] studied about a has a solution on Navier-Stokes's equations with their study approached a stretching surface of the boundary layer structure and redoubted as velocity of the surface is very less than the free stream velocity along with this an opposite boundary layer can also be formed. Abel et al. [8] analysed effect of non-uniform heat source on magneto-hydrodynamic heat transfer in a liquid film over unsteady stretching sheet. Yazdi et al. [9] studied the MHD slip flow over nonlinear permeable stretching surface in the presence of a chemical reaction, the slip flow is about the characteristic size of the flow regime which is very small or at the very low pressure. Kai [10] has been studied the magnetic-hydrodynamic stagnation point flow of a non-Newtonian (viscoelastic fluid) flow and heat transfer due to a flow stretching sheet and they had a conclusion that the effect of non-Newtonian flow heat transfer is better than the Newtonian flow and heat transfer. Mahantesh et al. [11] focused on MHD flow and heat transfer with partial slip over a stretching surface and they presented analytical solutions of for two cases namely PST and PHF, in terms of Kummer's function.

Natalia et al. [12] power law index flow and heat transfer with the non-linearly moving flat plate of the free stream slip velocity and they have given a particular attention on dual solutions (upper and lower branch solu- 
tions).Dessie and Kishan [13] considered MHD on boundary layer flow and heat transfer of a fluid with variable viscosity, porous medium, viscous dissipation and heat source/sink respectively. Mahantesh et al. [14] studied MHD flow and their importance in various fields of science and engineering applications. Hassan [15] considered the electrically conducting viscous boundary layer flow and heat transfer. Shen et al. [16] investigated magneto hydrodynamic mixed convection flow in the presence of stagnation point flow over a stretching sheet along with velocity slip. In the real world, we are depending upon various mechanical and non-mechanical things. Taufail and Ali [17] worked on properties of flow and heat transfer. Gireesha et al. [18] studied the magnetic heat transfer in dusty fluid on continuously stretching non-isothermal surface and studied effect of different parameters effects carried out. Zaidi and Din [19] considered convective heat transfer and importance of MHD effects on technology in various fields like automotive, aerospace and industry etc.

Abel et al. [20-25] investigated MHD flow and heat transfer effect on thermal boundary layer, hydromagnetic flow of viscoelastic liquid, heat transfer in a viscoelastic boundary layer flow, boundary layer flow and heat transfer characteristics of a second grade fluid and nonnewtonian fluid over a porous medium [24], MHD boundary layer flow and heat transfer characteristics of laminar liquid film [25] and continuously moving permeable stretching surface with non-uniform heat source/sink. Mahantesh et al. [26] worked on effects of thermal radiation [27], porous medium [28], heat transfer on nonlinearly stretching sheet with non uniform heat source and variable wall temperature [28], liquid film flow due to unsteady stretching sheet [29], MHD stagnation point of flow and heat transfer [30] and second order slip effects on flow [31]. Ibrahim and Haq [32] examined the convective heat transfer and magneto hydrodynamic stagnation point flow and they showed the thermal boundary layer thickness increases as the thermophoresis parameter decreases with the prandlt number Pr.

On observing all above investigations, there are no investigations on stagnation point flow of viscous fluid and heat transfer with velocity and thermal jump in presence of non-uniform heat source due to moving surface. Hence in the present study we have considered these effects on flow and heat transfer.

\section{Mathematical Formulation}

We have considered two dimensional steady, laminar flow and heat transfer of a incompressible fluid in the presence of transverse magnetic field strength $\mathrm{B}(\mathrm{x})$, which is effected normally on flow and given in the unique form as

$$
B(x)=B_{0} x^{\frac{n-1}{2}}, B_{0} \neq 0
$$

where $n$ is constant and $x$ is co-ordinate along the plate measured from the leading edge. The plate is moving inside or outside the origin with the velocity $u_{w}(x)=a x^{n}$ in an exterior (in viscid) flow of the velocity $u_{e}(x)=a x^{n}$, where $u$ and $v$ are the corresponding velocity components in the $x$ and $y$ directions respectively. Here $T_{w}(x)$ assumed as a temperature of the plate and ambient fluid is $T_{\infty}$ which constant temperature is.

The governing equations of continuity, momentum and energy equations are as follows,

$$
\begin{gathered}
\frac{\partial u}{\partial x}+\frac{\partial u}{\partial y}=0 \\
u \frac{\partial u}{\partial x}+v \frac{\partial u}{\partial y}=u_{e} \frac{d u_{e}}{d x}+v \frac{\partial^{2} u}{\partial y^{2}}-\frac{\sigma B^{2}(x)}{\rho} u \\
u \frac{\partial T}{\partial x}+v \frac{\partial T}{\partial y}=\alpha \frac{\partial^{2} T}{\partial y^{2}}+\frac{q^{\prime \prime \prime}}{\rho C p}
\end{gathered}
$$

where $\rho$ the electrical conductivity of the fluid, $\alpha$ is the thermal diffusivity, $u$ and $v$ are the velocity components $x$ and $y$ axis.

Associated with the initial and boundary conditions:

$$
\begin{aligned}
& u=v=0, T=T_{\infty} \text { for any } x, y \\
& v=0, u=a x^{n}+N_{1} v \frac{\partial u}{\partial y}, T=T_{w}(x)+S_{1} \frac{\partial T}{\partial y} \\
& \text { at } y=0, u-u_{e}(x)=a x^{n} \\
& T \rightarrow T_{\infty} \text { as } y \rightarrow \infty
\end{aligned}
$$

Here we guess the velocity slip factor $N_{1}$ and the temperature slip factor $S_{1}$ change with $x$ in the form $N_{1}=N x^{\left(\frac{1-n}{2}\right)}$ and $S_{1}=S x^{\left(\frac{1-n}{2}\right)}$ respectively, where $N$ and $S$ are positive slip constants. Furthermore expected that surface temperature is $T_{w}(x)=T_{\infty}+T_{0} x^{p}$ where $T_{0}$ the characteristics temperature parameter and $\mathrm{P}$ is the wall temperature parameter. It is concluded from $N_{1}=N x^{\left(\frac{1-n}{2}\right)}$ and $S_{1}=S x^{\left(\frac{1-n}{2}\right)}$ against physical point of view $n$ should be vary in the range $0 \leq n \leq 1$. If $n>1$ then $N_{1}$ and $S_{1}$ become singular at $x$ chose to the leading edge of the plate. It is remembered that boundary layer does not start at $\mathrm{x}=0$ but is starts in the vicinity of the leading edge of the plate [33]. Therefore the solution for $\mathrm{n}>1$ is realizable from the mathematical point of view. 
$C p$ is the specific heat at constant pressure, $\sigma$ is the electrical conductivity, $B_{0}$ is the applied magnetic field, $\mu$ is the viscosity, $\mathrm{T}$ is the temperature, $\mathrm{k}$ is the thermal conductivity of the fluid and $q^{\prime \prime \prime}$ is the rate of non-uniform heat generation/absorption coefficient and defined as

$$
q^{\prime \prime \prime}=\left(\frac{k u_{w}(x)}{x v}\right)\left[A^{\star}\left(T_{w}-T_{\infty}\right) f^{\prime}+B^{\star}\left(T-T_{\infty}\right)\right]
$$

Here $A^{\star}$ and $B^{\star}$ are the specifications of space and temperature dependent internal heat generation/absorption respectively. We can note that $A^{\star}>0 \& B^{\star}>0$ for the internal thermal generation and $A^{\star}<0 \& B^{\star}<0$ for the internal thermal absorption [28].

\section{Tranformation of PDE of Flow and Heat transfer equations to ODEs}

Using self-similar solution by means of the similarity function $f$ defined by

$$
\begin{aligned}
& u=a x^{n} f^{\prime}(\eta), \\
& v=-\sqrt{\frac{a v(n+1)}{2}} x^{\left(\frac{n-1}{2}\right)}\left[f(\eta)+\frac{n-1}{n+1} \eta f^{\prime}(\eta)\right]
\end{aligned}
$$

To solve Eqs. (2) to (4) with the boundary conditions (5) we use the following similarity variables

$$
\begin{aligned}
& \psi=\sqrt{\frac{2 a v}{n+1}} x^{\frac{n+1}{2}} f(\eta), \quad \theta(\eta)=\frac{T-T_{\infty}}{T_{w}-T_{\infty}}, \\
& \eta=\sqrt{\frac{a(n+1)}{2}} x^{\frac{n-1}{2}} y
\end{aligned}
$$

where $\psi$ is the stream function, which is defined as $u=\frac{\partial \psi}{\partial y}$ and $v=-\frac{\partial \psi}{\partial x}, v=\frac{\mu}{\rho}$ is the kinematic viscosity.

The fundamental partial differential Eqs. (3) and (4) converted to the ordinary differential equations by substituting Eqs. (6) - (8) in Eqs. (2) - (4), we obtain the following ordinary differential equations,

$$
\begin{gathered}
f^{\prime \prime \prime}+f f^{\prime \prime}+\left(\frac{2 n}{n+1}\right)\left[1-\left(f^{\prime}\right)^{2}\right]-M_{n} f^{\prime}=0 \\
\frac{1}{\operatorname{Pr}} \theta^{\prime \prime}+f \theta^{\prime}-\left(\frac{2 p}{n+1}\right) f^{\prime} \theta+\left(\frac{2}{n+1}\right) \frac{1}{\operatorname{Pr}}\left[A^{\star} f^{\prime}+B^{\star} \theta\right]=0
\end{gathered}
$$

where $\operatorname{Pr}=\frac{v}{\alpha}$ is the Prandtl number and the converted boundary conditions Eqs. (5) becomes,

$$
\left.\begin{array}{l}
f(0)=0, f^{\prime}(0)=\lambda+\beta f^{\prime \prime}(0), f^{\prime}(\infty)=1 \\
\theta(0)=1+\sigma \theta^{\prime}(0), \theta(\infty)=0
\end{array}\right\}
$$

where $\lambda=\frac{c}{a}$ is the moving parameter with $\lambda>0$ corresponding to downstream movement of the plate from the origin while $\lambda<0$ corresponding to the moving of the plate into the origin $\beta=N \sqrt{\frac{a v(n+1)}{2}}$ is the velocity slip parameter and $\sigma=S \sqrt{\frac{a(n+1)}{2 v}}$ is the temperature slip parameter. It is worth mentioning that $\mathrm{n}=1$ (stagnation point flow), $\mathrm{p}=0$ (isothermal plate) when $\beta=\lambda=\sigma=0$, Eqs. (9) and (10) along with the boundary conditions (11) become identical for $m=1$ (stagnation point flow and heat transfer) Bejan [34].

The physical quantities of the local skin friction coefficient $C_{f}$ and local Nusselt number $N u_{x}$ in this problem are defined as

$$
C_{f}=\frac{\tau_{w}}{\rho u_{e}^{2}(x)}, N u_{x}=\frac{x q_{w}}{k\left(T_{w}-T_{\infty}\right)}
$$

Where the skin friction (shear stress) along the plate is $\tau_{w}$ and wall heat $q_{w}$ are given by

$$
\tau_{w}=\mu\left(\frac{\partial u}{\partial y}\right), q_{w}=-k\left(\frac{\partial T}{\partial y}\right)_{y=0}
$$

Using the Eqs. (8),(12) and (13), we get

$$
\sqrt{\operatorname{Re}_{x}} C_{f}=\sqrt{\frac{n+1}{2}} f^{\prime \prime}(0), \operatorname{Re}_{x}^{-\frac{1}{2}} N u_{x}=\sqrt{\frac{n+1}{2}}\left[-\theta^{\prime}(0)\right]
$$

where $\operatorname{Re}_{x}=\frac{u_{e}(x) x}{v}$ is the Reynolds number.

\section{Procedure of Numerical computation}

The governing non-linear partial differential equations Eq. (3) and (4) are converted into non-linear ordinary differential equations Eq. (9) and (10). To find the solutions of Eqs. (9) and (10) we required five boundary conditions, three on equation of motion and two on equations of temperature respectively. But here $f^{\prime \prime}(\eta)$ and $\theta^{\prime}(\eta)$ are missing boundary conditions, hence solving the boundary value problem of Eq. (9) and (10) is difficulty, therefore, in the boundary conditions Eq. (11) we replace infinity to a finite value. The Eq. (9) and (10) with boundary conditions (11) are solved a numerical BVP method with efficient shooting technique and for the practical we considered the relative tolerance to $10^{-6}$. Finally, the converged result of BVP's are obtained. These all the above said procedure is implemented through the MAPLE13 computing software. 


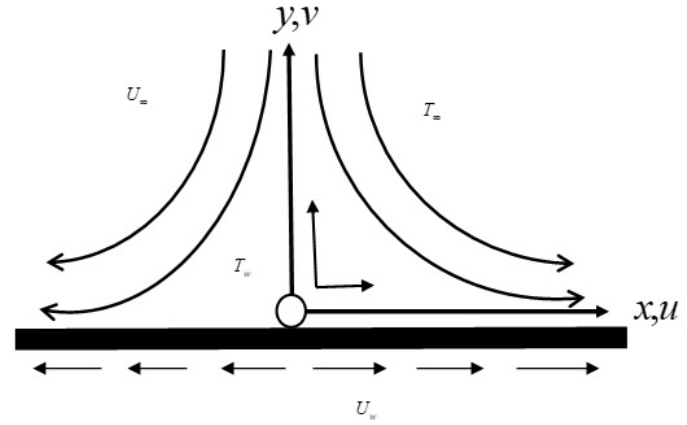

Fig. 1: Physical sketch of the stagnation point flow

\section{Results and Discussion}

We consider an analysis to study MHD slip flow and heat transfer in presence of stagnation point of a stretching sheet with non-linearly moving flat plat in a parallel free stream. The boundary layer equations of momentum and heat transfer are solved analytically and the different analytical expressions are obtained for non-dimensional temperature profile for different parameters. Numerical computations of results are demonstrated in the following figures. The characteristics of the parameters are studied in the present problem are power law index parameter $n$, wall temperature parameter $p$, magnetic field $\mathrm{Mn}$, prandlt number Pr, space-dependent heat source/sink parameter $A^{\star}$ and temperature dependent heat source/sink parameter $B^{\star}$. We now proceed with the discussion of results.

Fig. 1 Shows the physical phenomena of the considered problem.

Fig. 2 and 3 show the flow velocity profile respectively for the different values of $\lambda$ and $\beta$. It is observed that as increasing the value of $\lambda$ and $\beta$ which reduces the thickness of the boundary layer flow.

Fig. $4 \& 5$ are depicted for flow and velocity profile of different values of index law parameter $n$ (remaining parameters are remains same), as increasing power law index parameter $n$ both the flow and velocity increases, which intern increases the thickness of the momentum boundary layer.

Fig. 6 is demonstrated the velocity profile for different values of $M$, due the application of Lorentz force, the velocity of the boundary layer decreases as increases the $M$.

Fig. 7 and 8 plotted for the velocity profile for the different values of $\lambda$ and $\beta$. In this figures we observed that as

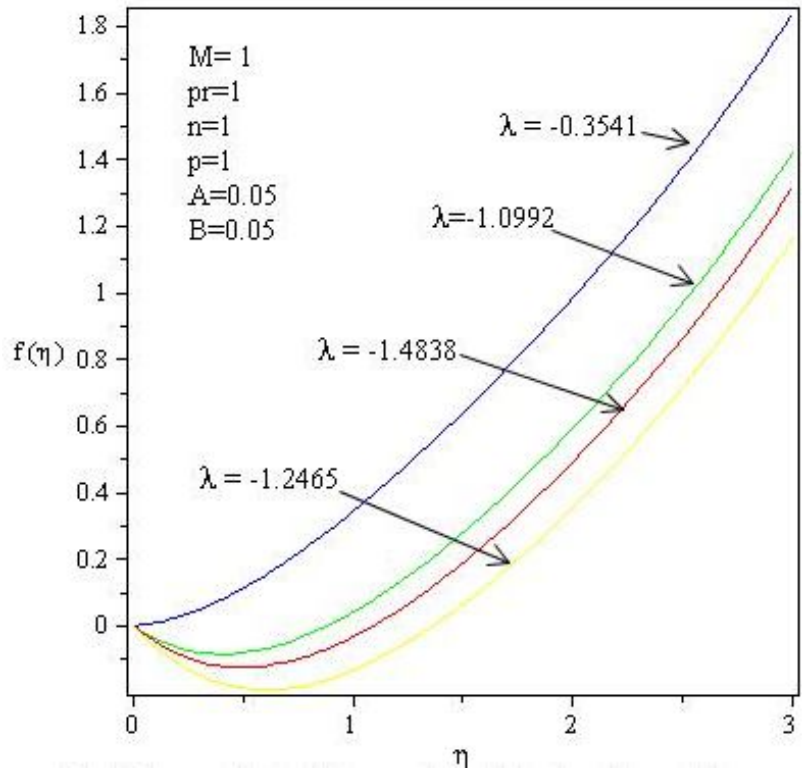

Fig. 2: Flow profile for different values of $\lambda$ when $\beta=\sigma=0.5$

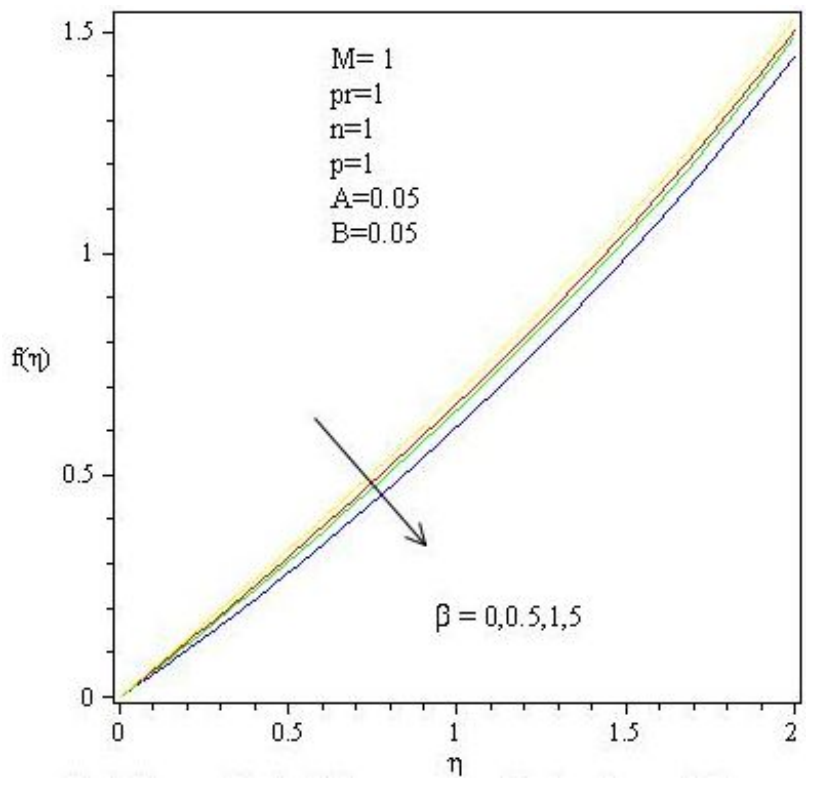

Fig. 3: Flow profile for different values of $\beta$ when $\lambda=\sigma=0.5$ 
Table 1: The comparison values of $-\theta^{\prime}(0)$ for different values of $\operatorname{Pr}$ when $n=1, p=0$ and $\beta=\lambda=\sigma=0$

\begin{tabular}{lccccc}
\hline \multicolumn{1}{c}{$\operatorname{Pr}$} & \multicolumn{5}{c}{} \\
\hline & 0.7 & 0.8 & 1 & 5 & 10 \\
\hline Present values & 0.49586 & 0.57046 & 0.57046 & 1.04343 & 1.33879 \\
Rosca et al. [12] & 0.49586 & 0.572274 & 0.57046 & 1.04343 & 1.33879 \\
Bejan [34] & 0.496 & 0.523 & 0.570 & 1.043 & 1.344 \\
\hline
\end{tabular}

Table 2: The Numerical values of Local Skin-Friction and Local Nusselt number

\begin{tabular}{|c|c|c|c|c|c|c|c|c|c|}
\hline$n$ & $M$ & $P$ & $A$ & $B$ & $\operatorname{Pr}$ & $f^{\prime \prime}(0)$ & $-\theta^{\prime}(0)$ & $\sqrt{\operatorname{Rex}_{e x}} C_{F}=\sqrt{\frac{n+1}{2}} f^{\prime \prime}(0)$ & $\mathrm{R}_{e x}^{\frac{-1}{2}} N u_{x}=-\sqrt{\frac{n+1}{2}} \theta^{\prime}(0)$ \\
\hline & 0.5 & & & & & 1.01523 & 0.68256 & 1.01523 & 0.68256 \\
\hline \multirow[t]{2}{*}{1} & 1.0 & 1.0 & 0.05 & 0.05 & 1 & 0.86116 & 0.62571 & 0.86116 & 0.62571 \\
\hline & $\begin{array}{l}1.5 \\
0.5\end{array}$ & & & & & $\begin{array}{l}0.75150 \\
1.13614\end{array}$ & $\begin{array}{l}0.57832 \\
0.67647\end{array}$ & $\begin{array}{l}0.75150 \\
1.27024\end{array}$ & $\begin{array}{l}0.57832 \\
0.75631\end{array}$ \\
\hline 1.5 & 1.0 & 1.0 & 0.05 & 0.05 & 1 & 0.98647 & 0.62813 & 1.10290 & 0.70226 \\
\hline 1.0 & 1.5 & & & & & $\begin{array}{l}0.87406 \\
1.01522\end{array}$ & $\begin{array}{l}0.58652 \\
0.68256\end{array}$ & $\begin{array}{l}0.97722 \\
1.01522\end{array}$ & $\begin{array}{l}0.65574 \\
0.68256\end{array}$ \\
\hline 1.5 & 0.5 & 1.0 & 0.05 & 0.05 & 1 & 1.13614 & 0.67647 & 1.27024 & 0.75631 \\
\hline 2.0 & & & -0.05 & & & $\begin{array}{l}1.21063 \\
1.13614\end{array}$ & $\begin{array}{l}0.66756 \\
0.73822\end{array}$ & $\begin{array}{l}1.71208 \\
1.27024\end{array}$ & $\begin{array}{l}0.94407 \\
0.82535\end{array}$ \\
\hline \multirow[t]{2}{*}{1.5} & 0.5 & 1.0 & 0.0 & 0.05 & 1 & 1.13614 & 0.70734 & 1.27024 & 0.79083 \\
\hline & & & 0.05 & -0.05 & & $\begin{array}{l}1.13614 \\
1.13614\end{array}$ & $\begin{array}{l}0.67647 \\
0.72266\end{array}$ & $\begin{array}{l}1.27024 \\
1.27024\end{array}$ & $\begin{array}{l}0.75632 \\
0.80795\end{array}$ \\
\hline \multirow[t]{2}{*}{1.5} & 0.5 & 1.0 & 0.05 & 0.0 & 1 & 1.13614 & 0.69981 & 1.27024 & 0.78240 \\
\hline & & 1 & & 0.05 & & $\begin{array}{l}1.13614 \\
1.13614\end{array}$ & $\begin{array}{l}0.67647 \\
0.67647\end{array}$ & $\begin{array}{l}1.27024 \\
1.27024\end{array}$ & $\begin{array}{l}0.75631 \\
0.75631\end{array}$ \\
\hline \multirow[t]{2}{*}{1.5} & 0.5 & 2 & 0.05 & 0.05 & 1 & 1.13614 & 0.82654 & 1.27024 & 0.92409 \\
\hline & & 3 & & & 1 & $\begin{array}{l}1.13614 \\
1.13614\end{array}$ & $\begin{array}{l}0.94171 \\
0.67647\end{array}$ & $\begin{array}{l}1.27024 \\
1.27024\end{array}$ & $\begin{array}{l}1.05286 \\
0.75631\end{array}$ \\
\hline \multirow[t]{2}{*}{1.5} & 0.5 & 1.0 & 0.05 & 0.05 & 2 & 1.13614 & 0.91117 & 1.27024 & 1.01871 \\
\hline & & & & & 3 & 1.13614 & 1.07204 & 1.27024 & 1.19857 \\
\hline
\end{tabular}

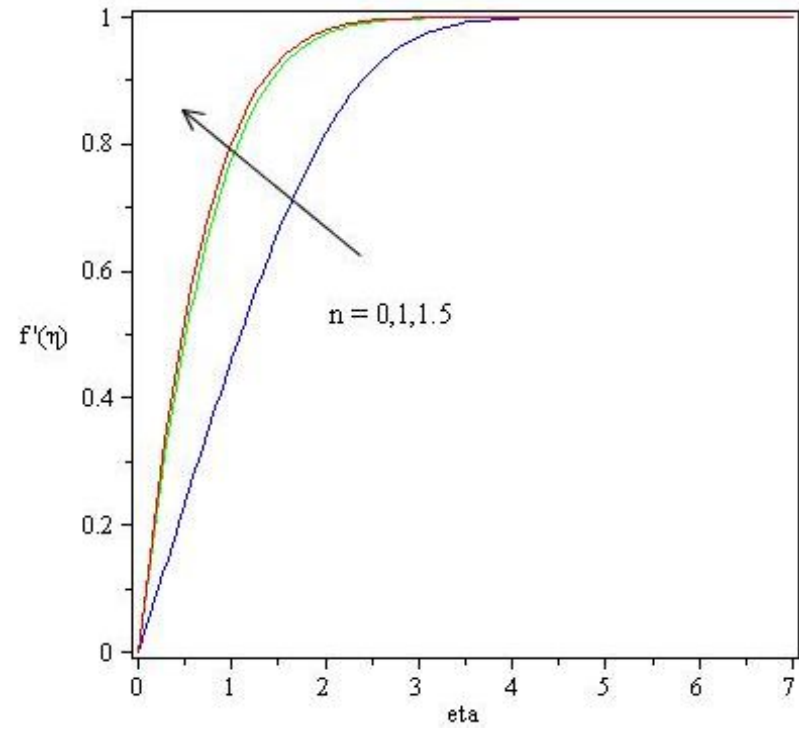

Fig. 4: Velocity profile for different values of $n$ and different parameter $p=1, p r=1, M=0, A=-0.05, B=-0.05$

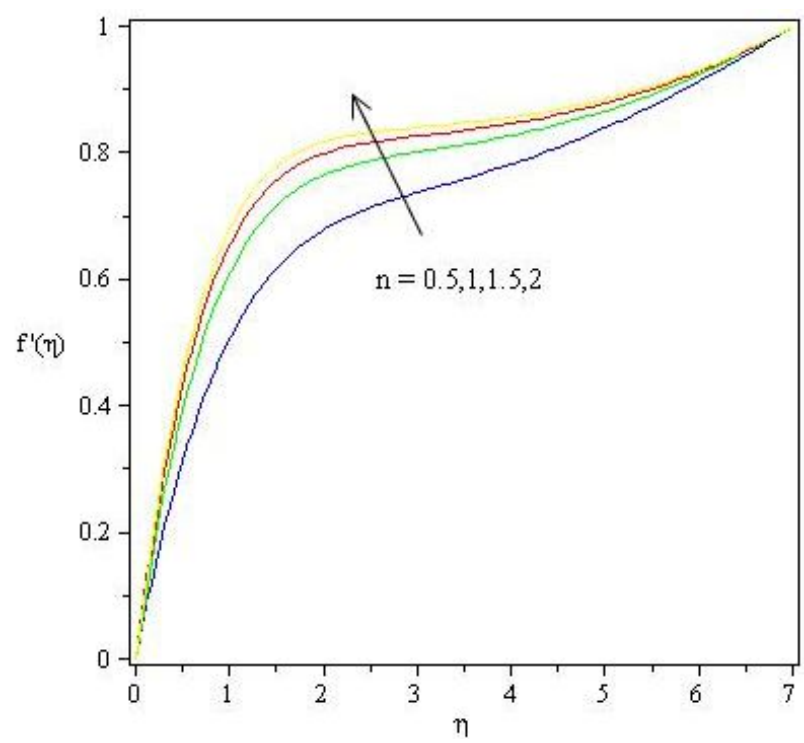

Fig. 5: Velocity profile for different values of $n$ and different parameter $p=1, p r=1, M=0.5, A=-0.05, B=-0.05$ 


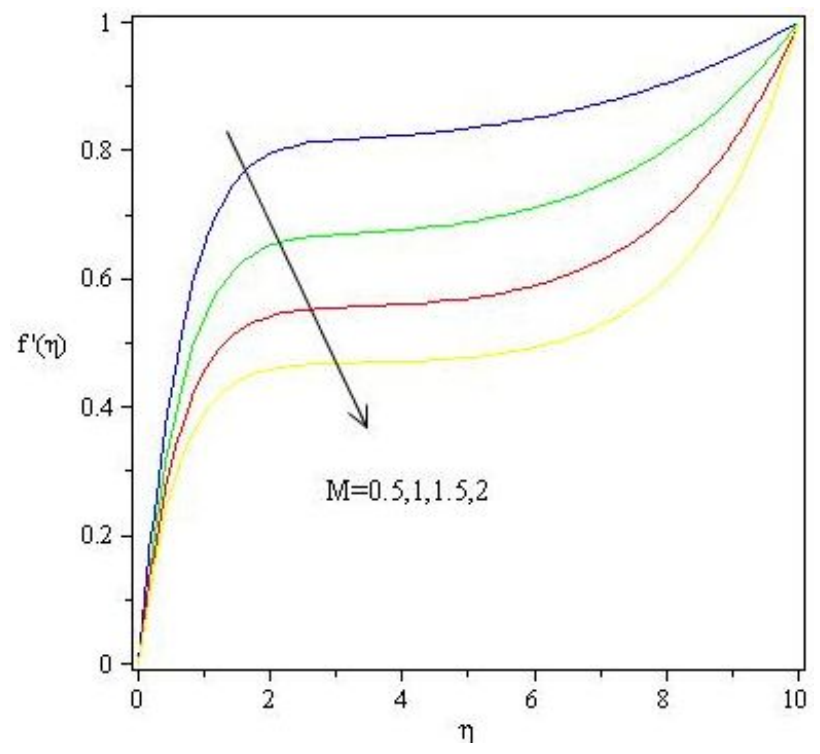

Fig. 6: Velocity profile for different values of $M$ and $n=1.5, P r=$ $1, A=0.5, B=0.5, p=1$

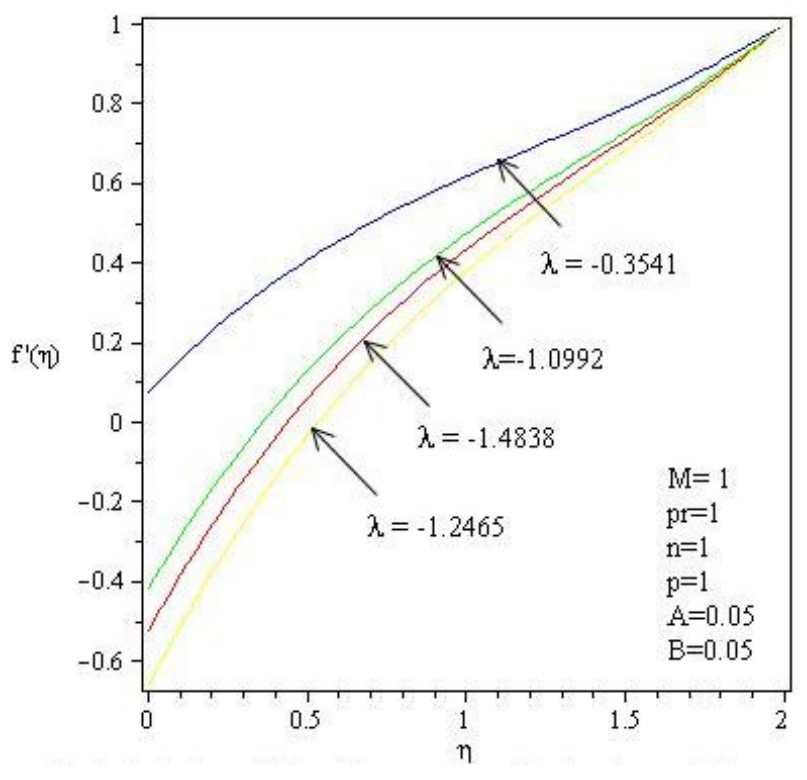

Fig. 7: Velocity profile for different values of $\lambda$ when $\beta=\sigma=0.5$

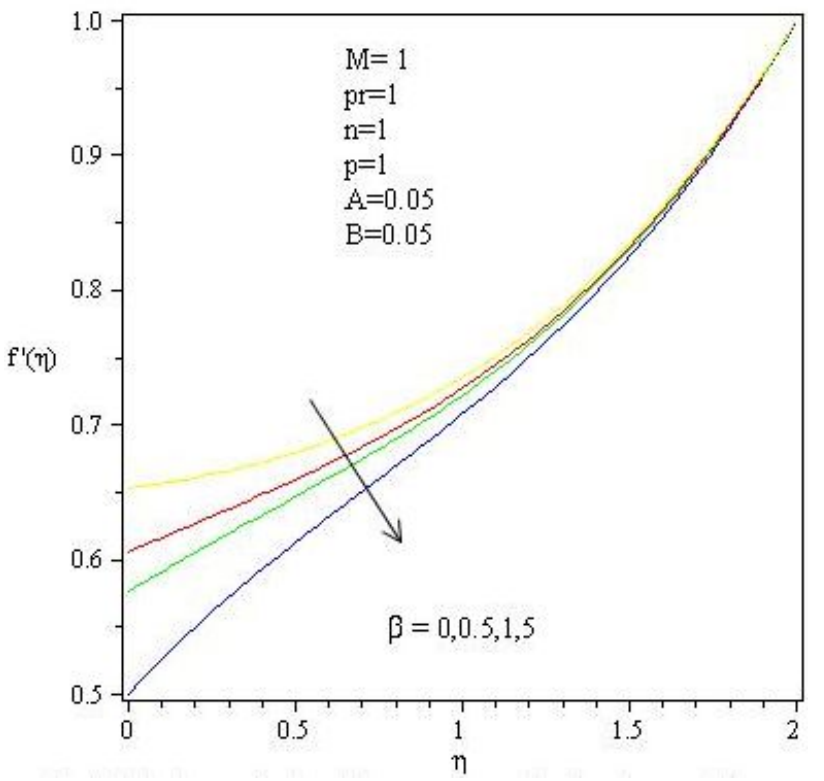

Fig. 8: Velocity profile for different values of $\beta$ when $\lambda=\sigma=0.5$

increasing the value of $\lambda$ it increases the thickness of the velocity but in the case of velocity slip parameter $\beta$ found that the opposite result.

Fig. 9 and 10 demonstrated the combined profile for different values of $n$ and $M$ and other physical parameters respectively. In both the profile, as increasing the values of $n$ and $M$ which increases the thickness of the velocity of the boundary layer flows respectively.

The Fig. 11 and 12 directed for the skin friction profile for various values of moving parameter $\lambda$ and velocity slip parameter $\beta$. Here the effects are found as, as increasing the values of moving parameter $\lambda$ which increases the thickness of the skin-friction of the boundary layer but decreases as increasing the velocity slip parameter $\beta$.

Fig. 13 \& 14. Presented the effect of physical parameter power law index $\mathrm{n}$ is depicted for temperature profile, which shows the result as increasing the non-linear stretching parameter $\mathrm{n}$ decreasing the temperature i.e., decreasing the thickness of the thermal boundary layer. Due to the Lorentz force, increases the thermal boundary layer thickness as increasing the magnetic field effects, hence the Fig. 15 plotted for the effect of magnetic field on temperature.

Fig. $16 \& 17$ shown for temperature profile for various values of prandlt number Pr, the characteristics of the parameter prandlt number shows that decreasing the thickness of the thermal boundary layer as increasing the Pr, hence decreasing the temperature due to the thermal layer.

Fig. 18 \& 19 presented temperature profile for different values of $A^{\star}$ and $B^{\star}$ which are called space-dependent heat 


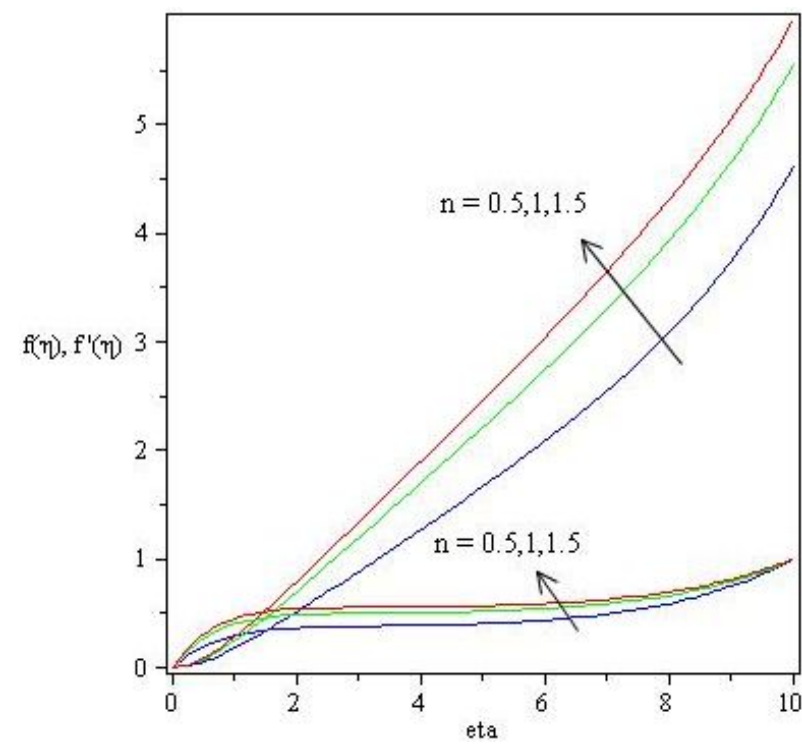

Fig. 9: Combined profile of power law index for flow and velocity for different values of $n$ and $p=1, M=1.5, \operatorname{Pr}=1, A=0.5, B=0.5$

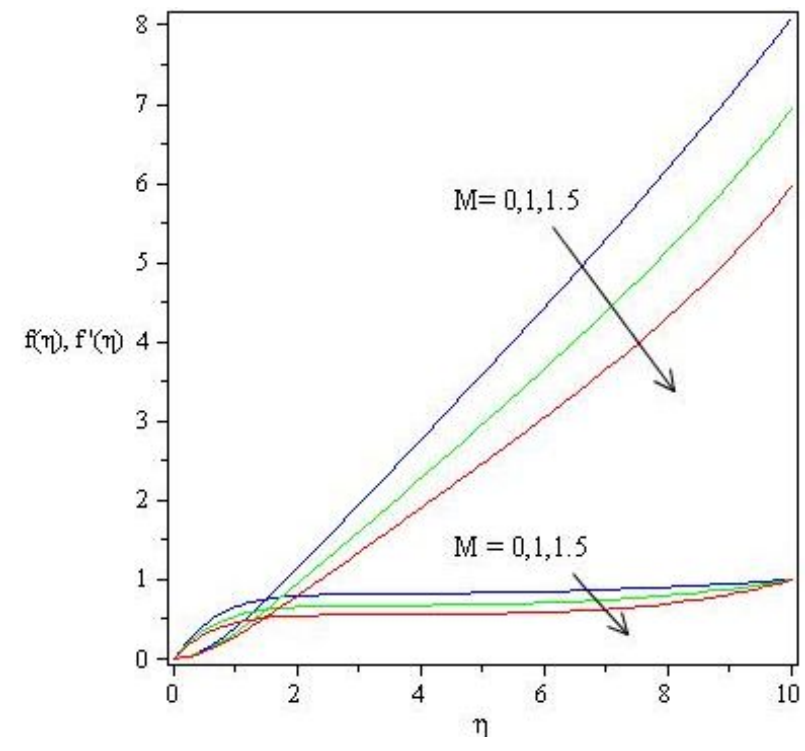

Fig. 10: Combined profile of flow and velocity for different values of $M$ and $p=1, M=1.5, P r=1, A=0.5, B=0.5, n=1$

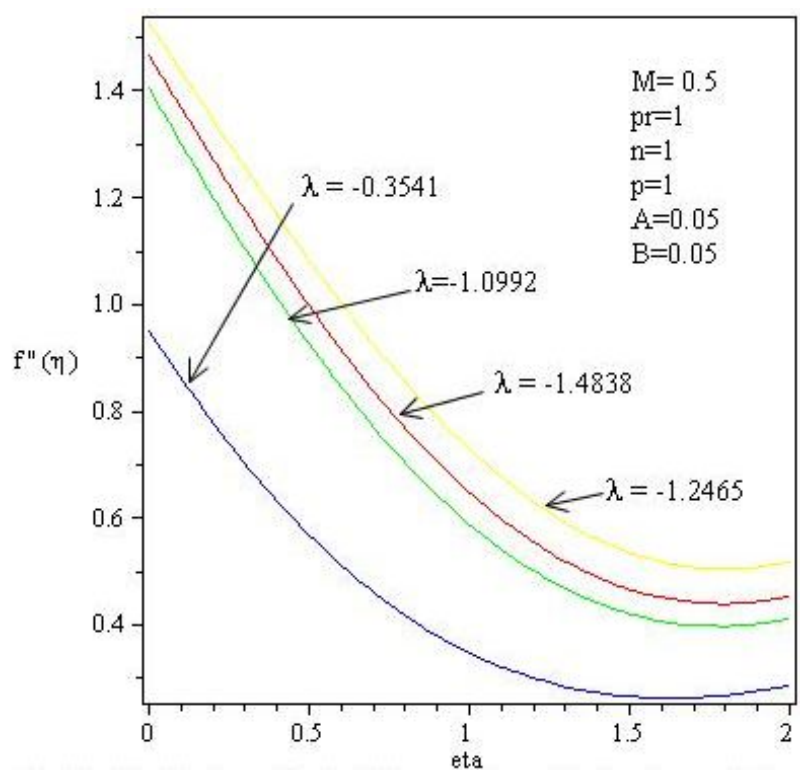

Fig. 11: Skin-friction profile for different values of $\lambda$ when $\beta=\sigma=$ 0.5

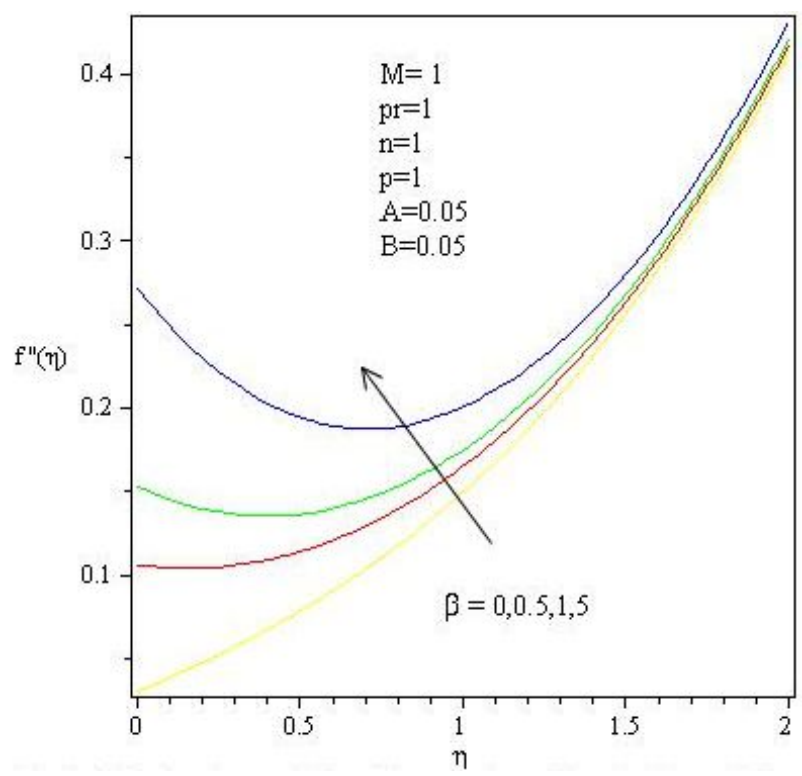

Fig. 12: Skin-friction profile for different values of $\beta$ when $\lambda=\sigma=$ 0.5 


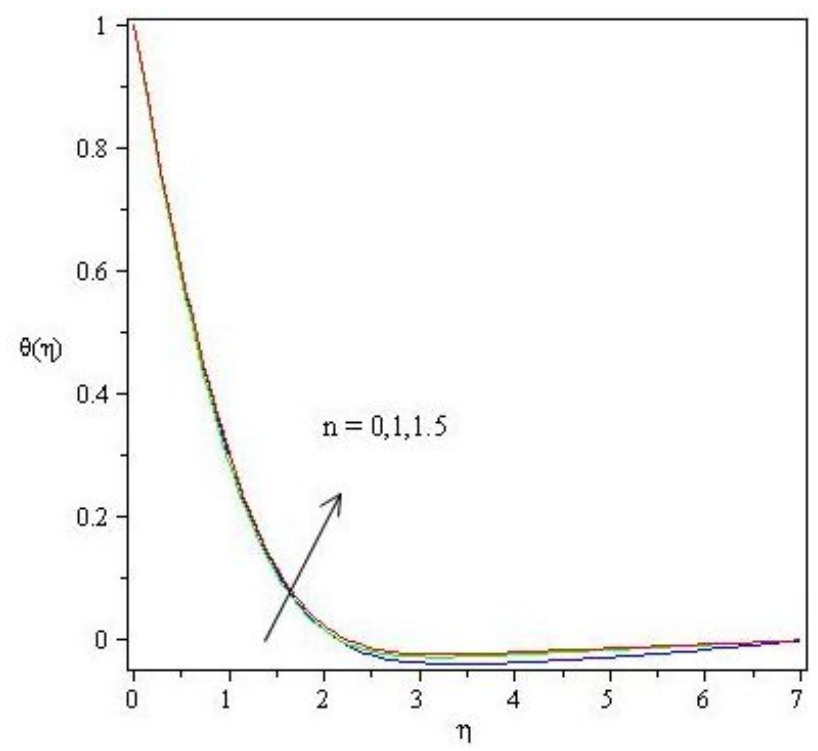

Fig. 13: Temperature profile for different values of $n$ and different parameters $p=1, M=0, A=-0.05, B=-0.05, p r=1$

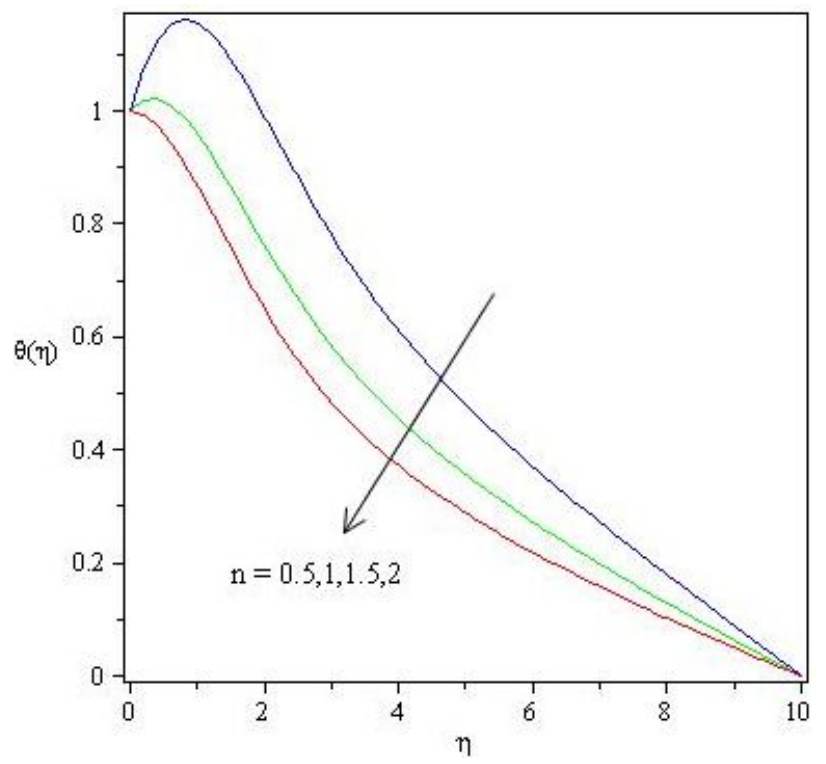

Fig. 14: Temperature profile for different values of $n$ and different parameters $p=1, p r=1, M=0.5, A=0.5, B=0.5$

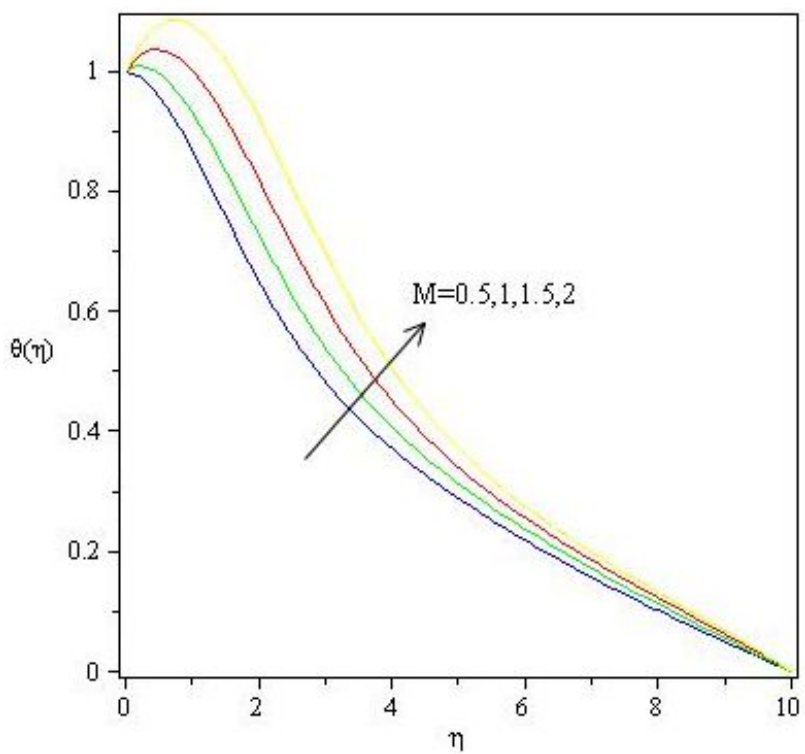

Fig. 15: Temperature profile for different values of $M$ and $n=$ 1.5, $p=1, A=0.5, B=0.5, p r=1$

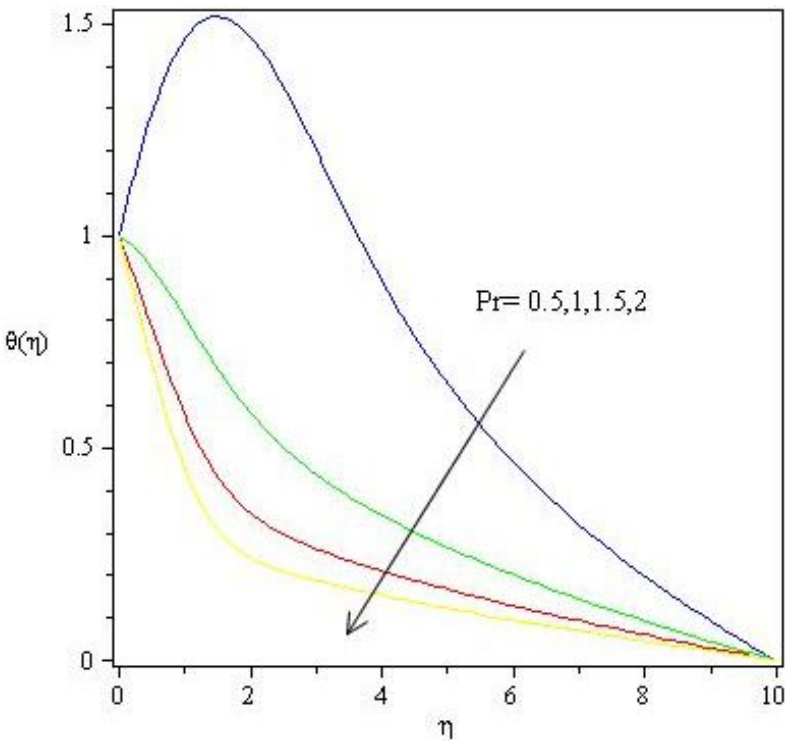

Fig. 16: Temperature profile for different values of $\operatorname{Pr}$ and $M=0, p=$ $1, n=1.5, A=0.5, B=0.5$ 


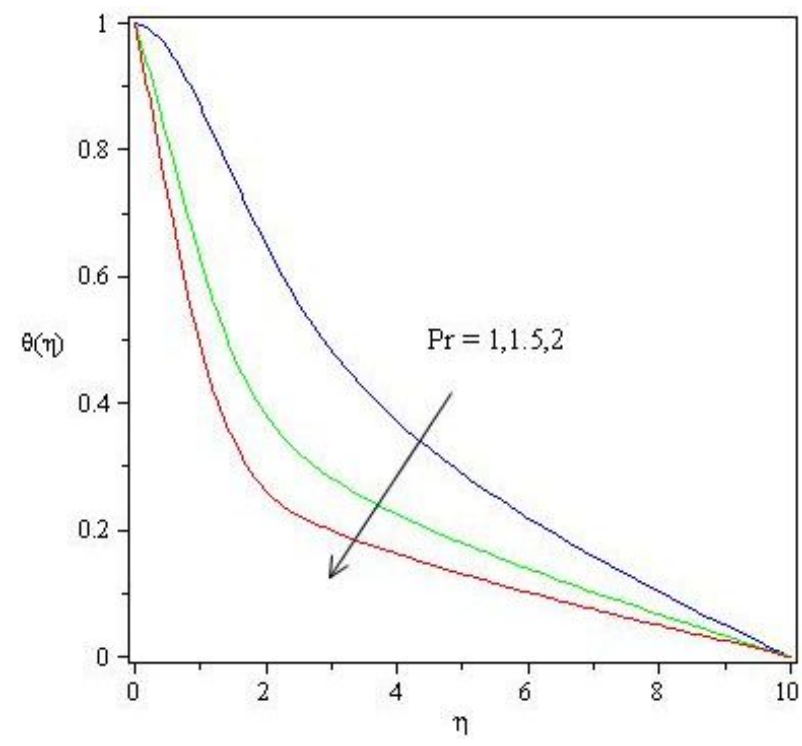

Fig. 17: Temperature profile for different values of $\operatorname{Pr}$ and $M=$ $0.5, p=1, n=1.5, A=0.5, B=0.5$

source/sink parameter and temperature dependent heat source/sink parameter. And Fig. 20 shows the profile for wall temperature parameter as increasing the power law index $\mathrm{p}$ which reduces the temperature in the boundary layer.

Fig. 21 and 22 plotted for temperature profile different values of moving parameter $\lambda$ and velocity slip parameter $\beta$. As increasing the moving parameter $\lambda$ which increases the thickness of the thermal boundary layer and velocity slip parameter $\beta$ also shows that the same effects of thermal boundary layer.

Fig. 23, 24 and 25 directed for the temperature gradient profile for various values of the physical parameters such as moving parameter, velocity slip and temperature slip parameters respectively. Here some points of effects of these physical parameters are found as, as increasing the values of moving parameter and velocity slip parameter the temperature gradient increases and temperature gradient decreases as increasing the temperature velocity parameter.

\section{Conclusions}

The problem of the stagnation point of MHD fluid flow and heat transfer has been studied in the presence of slip velocity, thermal jump and non-uniform heat source/sink. The numerical results are presented to analyze the various parameter characteristics. Among these results we found some important notices as follows

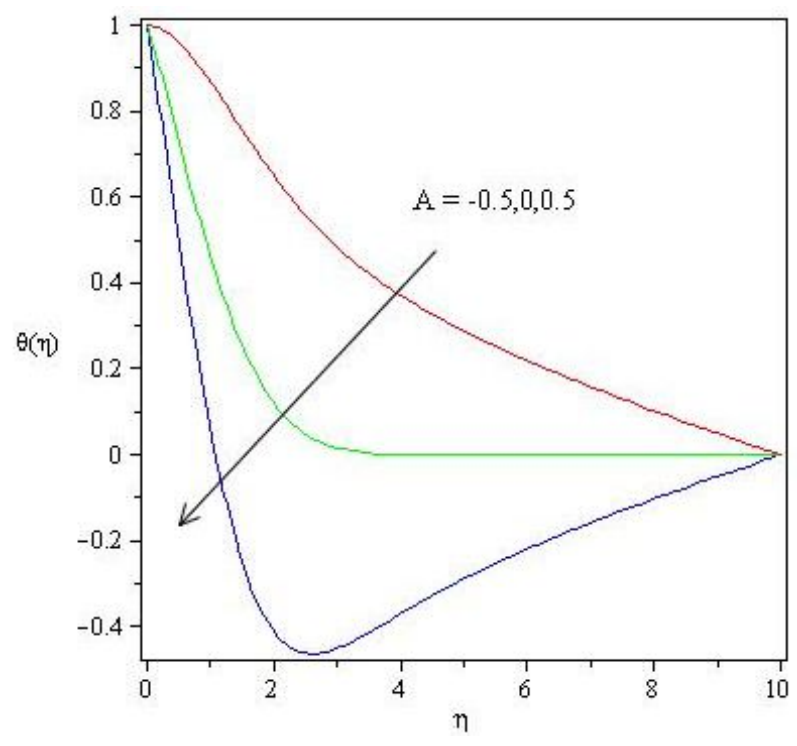

Fig. 18: Temperature profile for different values of $A=-0.5,0.5,1$ and $p=1, p r=1, M=0.5, B=0.5, n 1.5$

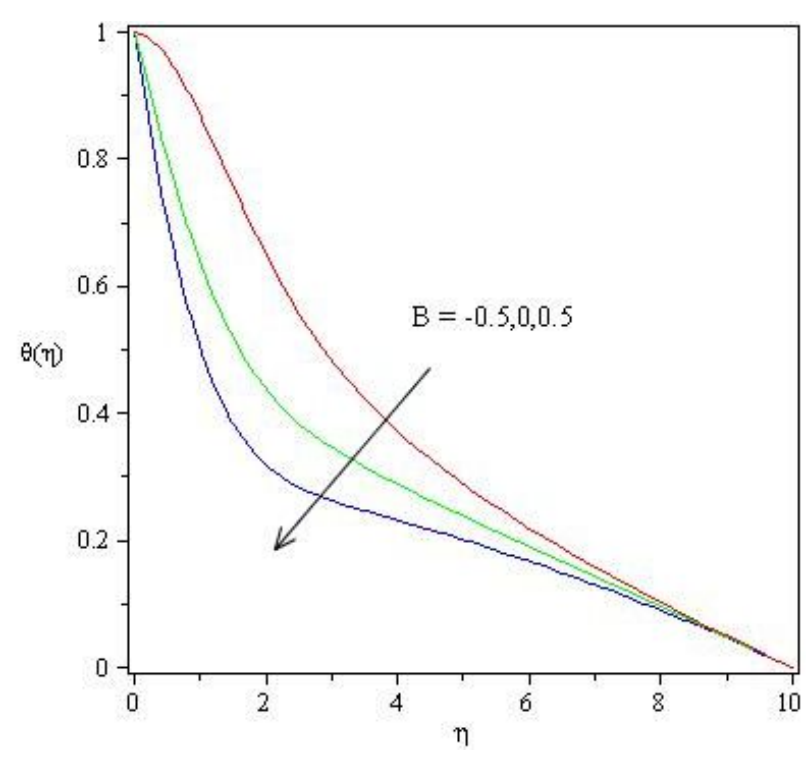

Fig. 19: Temperature profile for different values of $B=$ $-0.5,0,0.5,1$ and $p=1, p r=1, M=0.5, B=0.5, n=1.5$ 


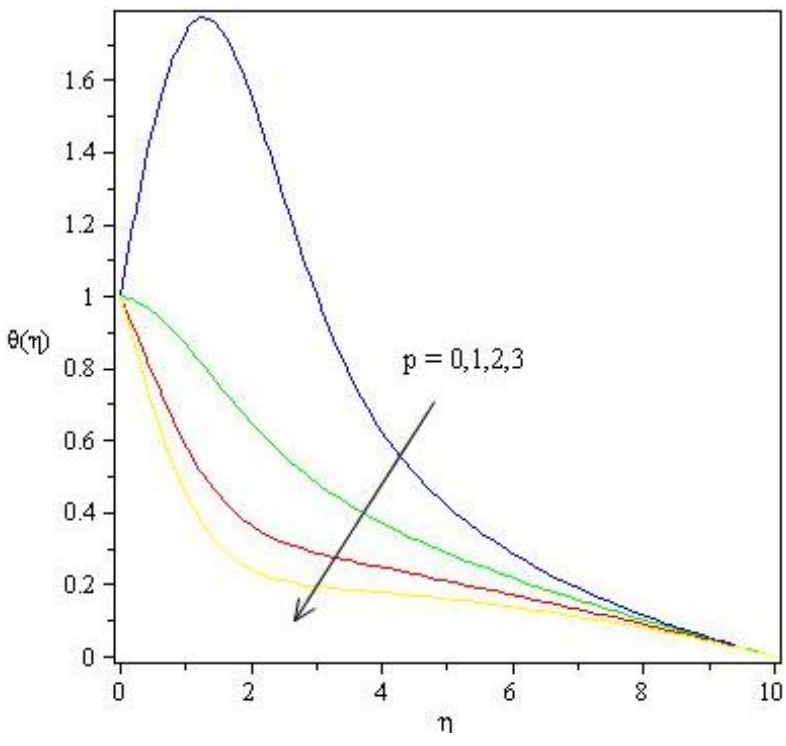

Fig. 20: Temperature profile for different values of power law index $p=0,1,2,3$ and $p r=1, M=0.5, A=0.5, B=0.5, n=1.5$

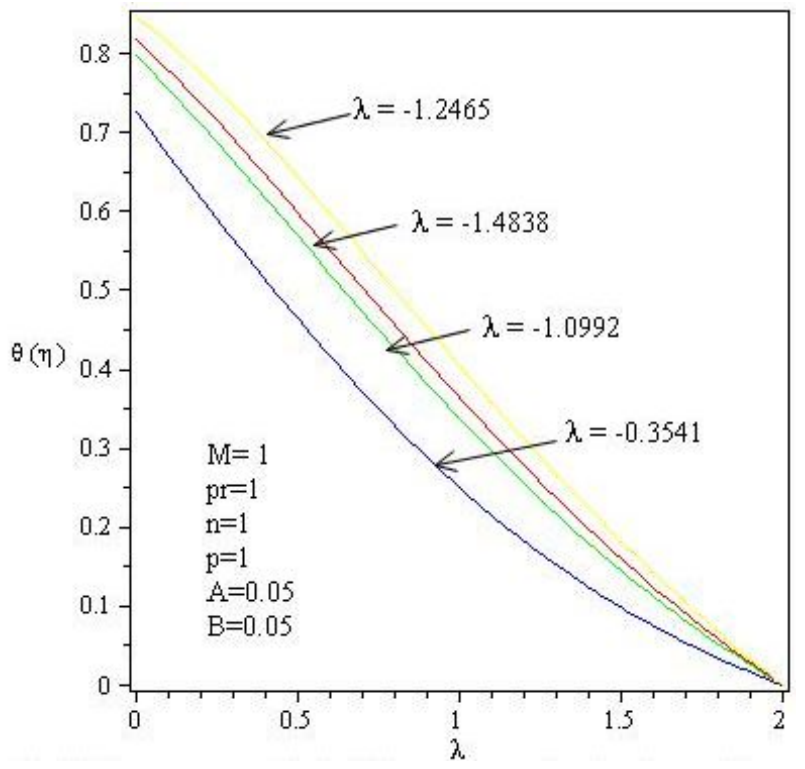

Fig. 21: Temperature profile for different values of $\lambda$ when $\beta=\sigma=$ 0.5

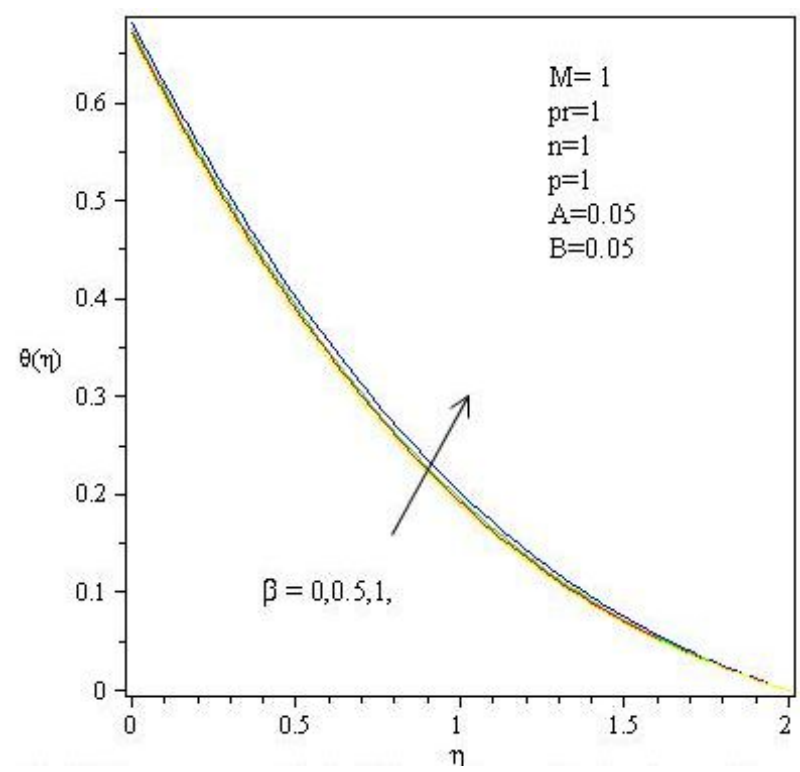

Fig. 22: Temperature profile for different values of $\beta$ when $\lambda=\sigma=$ 0.5

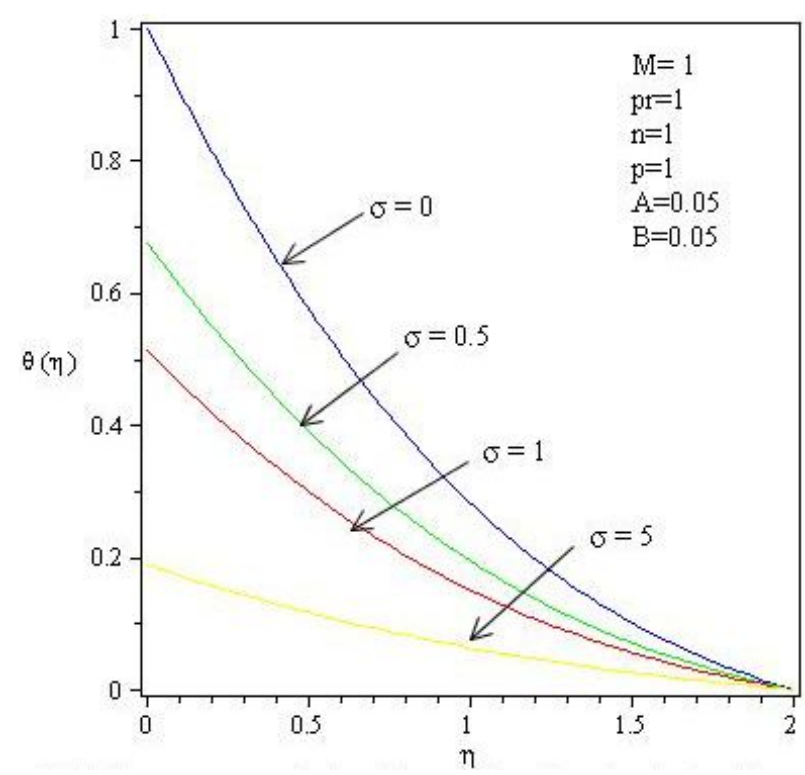

Fig. 23: Temperature profile for different values of $\sigma$ when $\beta=\lambda=$ 0.5 


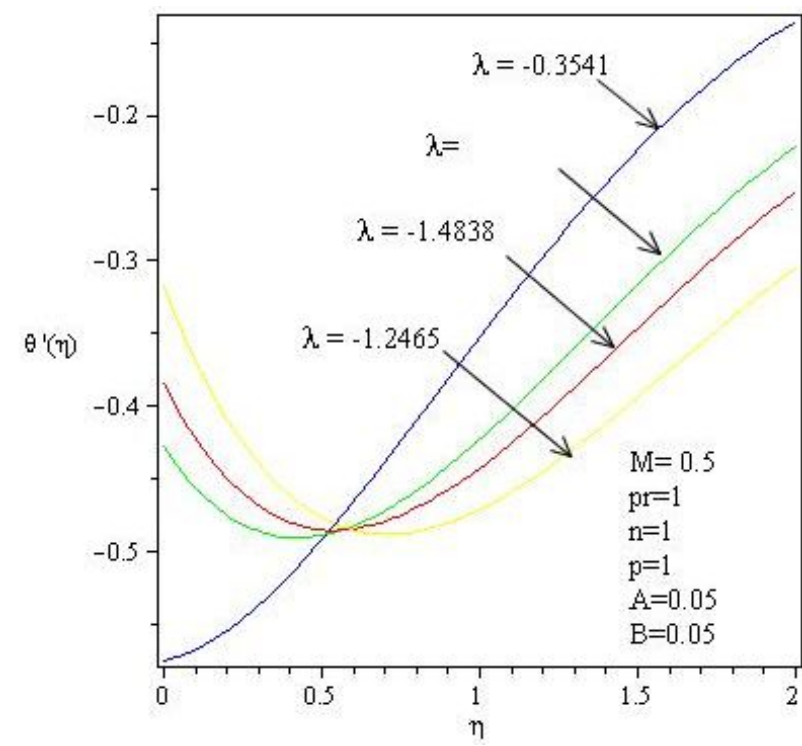

Fig. 24: Temperature gradient profile for different values of $\lambda$ when $\beta=\sigma 0.5$

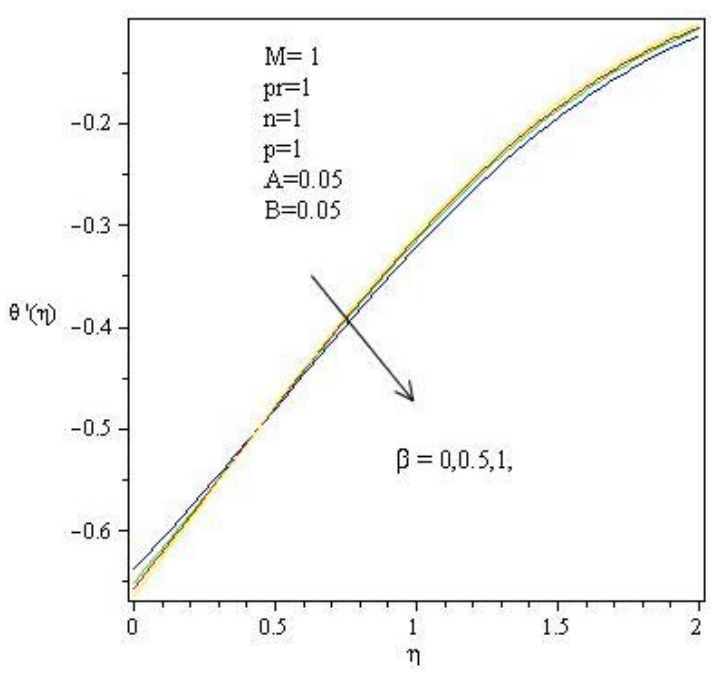

Fig. 25: Temperature gradient profile for different values of $\beta$ when $\lambda=\sigma=0.5$
- As increasing the power law index parameter which increases the velocity where as opposite result observed in the case of magnetic field

- The velocity increases as increasing the power law index in both the cases i.e., the magnetic field presents and absent.

- The temperature increases as increasing the power law index when magnetic field is absent and decreases when magnetic field is present.

- Whether the magnetic field is present or absent, the temperature decreases as increasing the Prandtl number.

- The temperature decreases as increasing the parameters $\mathrm{A}, \mathrm{B}$ and $\mathrm{p}$ in presence of magnetic field but increases in case of variation of magnetic field (see Fig. 15).

- The moving parameter $\lambda$ reduces the flow, velocity and temperature gradient and decreases the skin-friction as well as thermal boundary layer.

- The velocity slip parameter reduces the flow, velocity and skin-friction of the boundary layer but increases the thermal boundary layer and temperature gradient.

- The temperatures slip parameter effects only on thermal boundary layers and which reduces the thermal boundary layer where as decreases the temperature gradient.

\section{Nomenclature:}

$C_{p}$ Specific heat

$C_{f}$ Friction co-efficient

$f$ Dimensionless stream function

$K$ Thermal conductivity

$N u_{x}$ Local Nusselt number

Pr Prandlt number

$q_{w}$ Wall heat flux

$\mathrm{Re}_{x}$ Local Reynolds number

$N u_{x}$ Local nusselt number

$T$ Local fluid temperature

$T_{\infty}$ Free stream temperature

$T_{0}$ Characteristics temperature

$T_{w}$ Wall temperature

$u$ Velocity along $x$-axis

$u_{w}$ Velocity at wall

$v$ Velocity along y-axis

$x$ Distance along the plate

$y$ Distance normal to plate

$q^{\prime \prime \prime}$ Non-uniform heat source/sink

$B_{0}$ Applied magnetic field

$A^{\star}$ Space dependent internal heat generation

$B^{\star}$ Temperature dependent internal heat absorption

$\mathrm{P}$ Wall temperature parameter 
Mn Magnetic parameter

n Non-linear stretching parameter

\section{Greek symbol:}

$\psi$ Stream function

$\beta$ Velocity slip parameter

$\lambda$ Thermal jump parameter

$\sigma$ Temperature slip parameter

$\alpha$ Thermal diffusivity

$\eta$ Similarity variable

$\mu$ Dynamical viscosity

$\theta$ Dimensional temperature

$v$ Kinematic viscosity

$\rho$ Density

\section{References}

[1] K.Kanaka Raju, N. Muthiyalu, G. Venkateshwara Rao, Least square finite element solution of stagnation point flow, complex and fluids, 4(1976)143-147.

[2] Rama Subba Reddy Gorla, Non-Newtonian fluid at a stagnation point in the presence of a transverse magnetic field, Mch.Res.Comm,3(1976)1-6.

[3] H.S. Takhar, C.D. Surma Devi, G.Nath, MHD unsteady incompressible three dimensional asymmetric stagnation point boundary layers, 14(1)(1987) 29-35.

[4] Bruno Philip Besser, Richard Philip Rijnbeek, Helfried Karl biernat, Planar MHD stagnation point flows with velocity shear, planet, space sci,38(3)(1990)411-418.

[5] M.Massoudi, M.Ramezant, Heat transverse analysis of a viscoelastic fluid at a stagnation point, Mechanics Research communications, 19(2) (1992)129-134.

[6] P.Donald Ariel, three dimensional stagnation point flow of a viscoelastic fluid, Mechanics Research communications, 21(4)(1994)389-396.

[7] Tapas R.Mahapatra, Anadi S.Gupta, stagnation point flow towards a stretching surface, Canadian journal of chemical engineering, 81(2003)258-263.

[8] M. Subhas able, Jagadish Tawade, Mahantesh M.N, effect of non-uniform heat source on MHD heat transfer in a liquid film over an unsteady stretching sheet. International journal of non-linear Mechanics 44(2009)990-998.

[9] M.H.Yazdi, S.Abdullah, I.Hashim Sopian, slip MHD flow over permeable stretching surface with chemical reaction, $17^{\text {th }}$ Australian fluid mechanics conference, Auckhand, Newzealand 5-9 December 2010.

[10] Kai-Long Hsiao, MHD Stagnation Point Viscoelastic Fluid Flow And Heat Transfer On A Thermal Forming Stretching Sheet With Viscous Dissipation, the Canadian journal of chemical engineering, 89(2011)1228-1235.

[11] M.M. Nandeppanavar, K. Vajravelu, M. Subhas Abel, M.N. Siddalingappa, MHD flow and heat transfer over a stretching surface with variable thermal conductivity and partial slip, Meccanica 48(2013)1451-1464.

[12] Natalia C. Rosca, Alin V. Rosca, Ioan pop, stagnation point flow and heat transfer over a non-linearly moving flat plate in a parallel free stream with slip. Commun nonlinear sci number simulate 19(2014)1822-1835.

[13] H.Dessie, N.Kishan, MHD effects on heat transfer over stretching sheet embedded in porous medium with variable viscosity viscous dissipation and heat source/sink, A. S.Eng.J 5(2014)967-977.

[14] Mahantesh.M.Nandeppanavar, K.Vajravelu, P.S.Datti, optimal homotopy asymptotic solutions for non-linear ordinary differential equations arising in flow and heat transfer due to no-linear stretching sheet, Heat transfer -Asian Research,00(00)2014.

[15] Hossam S. Hassan, symmetry analysis for MHD viscous flow and heat transfer over a stretching sheet, Applied Mathematics, 6(2015)78-94.

[16] Ming Shen, Fei Wang, Hui Chen, MHD mixed convection slip flow near a stagnation point on a non linearly vertical stretching sheet, shen et.al, Boundary value problems 2015(2015)78.

[17] M.N.Tufail, A.Ali, Irreversibility analysis of MHD flow over an exponentially stretching sheet, heat transfer-Asian Research 44(3) 2015.

[18] B.J.Gireesha, B.Mahantesh, R.S.R.Gorla, P.T.Manjunatha, Thermal radiation and hall effects on boundary layer flow past a nonisothermal stretching surface embedded in porous medium with nonuniform heat source and fluid particle suspension, Heat Mass Transfer 52(2016) 897-911.

[19] Syed Zulfiquar ali Zaidi, Syed Tauseef Mohyud-din, convective heat transfer and MHd effects on two dimensional wall jet flow of a nanofluid with passive control model, Aerospace Science and Technology49(2016)225-230.

[20] M.S.Abel, E.Sanjayanand, M.M.Nandeppanavar, viscoelastic MHD flow and heat transfer over a stretching sheet with viscous and ohmic dissipations. Commun. Nonlinear Sci Numer Simulate 13 (2008) 1808-1821

[21] M. S.Abel, M.M.Nandeppanavar, heat transfer in Magneto hydrodynamic viscoelastic boundary layer flow over a stretching sheet with non-uniform heat source/sink, Commun. Nonlinear Sci Numer Simulate 14 (2009) 2120-2131.

[22] M.S.Abel, P.G.Siddheshwar, M.M.Nandeppanavar, heat transfer in a viscoelastic boundary layer flow over a stretching sheet with viscous dissipation and nonuniform heat source. Int.J. Heat Mass Transfer 50 (2007) 960-966.

[23] M.S.Abel, M.M.Nandeppanavar, S.B. Malipatil, heat transfer in a second grade fluid through a porous medium from a permeable stretching sheet with non-uniform heat source/sink, Int.J.Heat Mass Transfer 53 (2010) 1788-1795.

[24] M. Subhas Abel, J.Tawade, M.M.Nandeppanavar, effect of nonuniform heat source on magneto hydrodynamic heat transfer in a liquid film over an unsteady stretching sheet,Int.J.nonlinear mechanics 44(2009)990-998.

[25] M.S.Abel, M.M.Nandeppanavar \& M.B.Malkhed, hydromagnetic boundary layer flow and heat transfer in viscoelastic fluid over a continuously moving permeable stretching surface with non uniform heat source/sink embedded in fluidsaturated porous medium, Chem.Eng.Commun,197(2010)633-655.

[26] M.M.Nandeppanavar, K.Vajravelu, M.S.Abel, heat transfer in magneto hydrodynamic viscoelastic boundary layer flow over a stretching sheet with thermal radiation and nonuniform heat source/sink, Commun. Nonlinear Sci Numer Simulate 16 (2011) 3578-3590. 
[27] M.M.Nandeppanavar, M.S. Abel, K. Vajravelu, flow and heat transfer characteristics of a viscoelastic fluid in a porous medium over an impermeable stretching sheet with viscous dissipation, Int. J. Heat Mass Transfer 53 (2010) 4707-4713.

[28] Mahantesh M. Nandeppanavar, K. Vajravelu, M. Subhas Abel, Chiu-On Ng, Heat transfer over a nonlinearly stretching sheet with non-uniform heat source and variable wall temperature, International Journal of Heat and Mass Transfer 54 (2011) 4960-4965.

[29] Mahantesh M. Nandeppanavar, K. Vajravelu, M. Subhas Abel, S. Ravi, H. Jyoti, Heat transfer in a liquid film over an unsteady stretching sheet, International Journal of Heat and Mass Transfer 55 (2012) 1316-1324.

[30] Wubshet Ibrahim, Bandari Shankar, M.M.Nandeppanavar, magneto hydrodynamic stagnation point flow and heat transfer due to nanofluid towards a stretching sheet, Int. J. Heat Mass Transfer 56 (2013) 1-9.
[31] Mahantesh M. Nandeppanavar, K. Vajravelu, M. Subhas Abel, M.N. Siddalingappa, second order slip flow and heat transfer over a stretching sheet with nonlinear navier boundary condition, International Journal of Thermal Sciences 58 (2012) 143-150.

[32] W.Ibrahim, R.U.Haq, Magneto-hydrodynamic stagnation point flow of nanofluid past a stretching sheet with convective boundary condition, Braz.Soc.Mech.Sci.Eng.38 (2016)11551164.

[33] Martynenko OG, Khramtsov PP. Free-convective heat transfer. Berlin: Springer Verlag 2005.

[34] Bejan A. Convection heat transfer. 2nd ed. New York: Wiley; 1995. 